\title{
Framework for climate services in developing countries
}

\author{
F. H. M. Semazzi* \\ Department of Marine, Earth \& Atmospheric Sciences, North Carolina State University, Box 8208, Raleigh, \\ North Carolina 27695, USA
}

\begin{abstract}
An embryonic framework now exists for the provision of climate services in developing countries via the Regional Climate Outlook Forum supported by the World Meteorological Organization (WMO) Climate Information and Prediction Services (CLIPS) project. However, in general, the major advances in climate science research made during recent decades have hardly improved climate services or socio-economic conditions. It is therefore important, while the Global Framework for Climate Services (GFCS) is being developed, to examine the reasons for these shortcomings and formulate the appropriate mechanisms to address this problem. Although the lack of financial and technological resources, and the necessary infrastructure are critical drawbacks to addressing the climate change problem, we believe the major obstacle is the existence of a self-sustaining cycle, which is continuously eroding and undermining progress. This cycle begins with poor support and funding for research and climate services, leading to the inability of climate service providers to meet stakeholder needs and, thus, further eroding support and perpetuating the cycle. We propose an alternative approach that would break this cycle and create a new class of stakeholders for climate services in developing countries, who will use and advocate for funding and other forms of support for relevant research in the field and its outcomes. We propose a framework for the development of climate risk management strategies (CRMS) and climate services in developing countries. This framework comprises 3 components, namely, implementation sites for the CRMS incubation projects, a joint task force of Climate Variability and Predictability (CLIVAR) and CLIPS for guiding the relevant cross-cut research, and a WMO-World Climate Research Program mechanism for coordinating the CRMS agenda.
\end{abstract}

KEY WORDS: Climate services · CLIVAR · CLIPS · CRMS · GFCS

\section{INTRODUCTION}

The Global Framework for Climate Services (GFCS), which is in the process of being established, promotes the integration of climate information into developmental planning to enhance the performance of climate-sensitive socio-economic sectors (GFCS 2010). The problem regarding the provision of climate services in developing nations is compounded by inadequate funding to employ staff, the lack of technological resources and an insufficient infrastructure to support in-country climate services. An embryonic framework exists through the activities of the Regional Climate Outlook Forum (RCOF; RCOF 2008). RCOFs (see Table 1, for list of acronyms) are run with the help of the World Meteorological Organization (WMO), and other agencies in developing countries (Fig. 1) where they could play a key role in supporting the GFCS. They are an innovative concept conceived, developed and supported as part of WMO's Climate Information and Prediction Services (CLIPS; CLIPS 2010) project in partnership with the National Meteorological and Hydrological Services (NMHSs), regional climate institutions and other agencies. Despite the challenges posed by resources and human and infrastructural capacities, some of the RCOFs have achieved remarkable progress in their regional networking and user liaison functions, and have substantially contributed to capacity building and user awareness.

RCOFs receive their technical guidance primarily from the Commission for Climatology (CCL) of the WMO through its CLIPS (CLIPS 2010) project. 


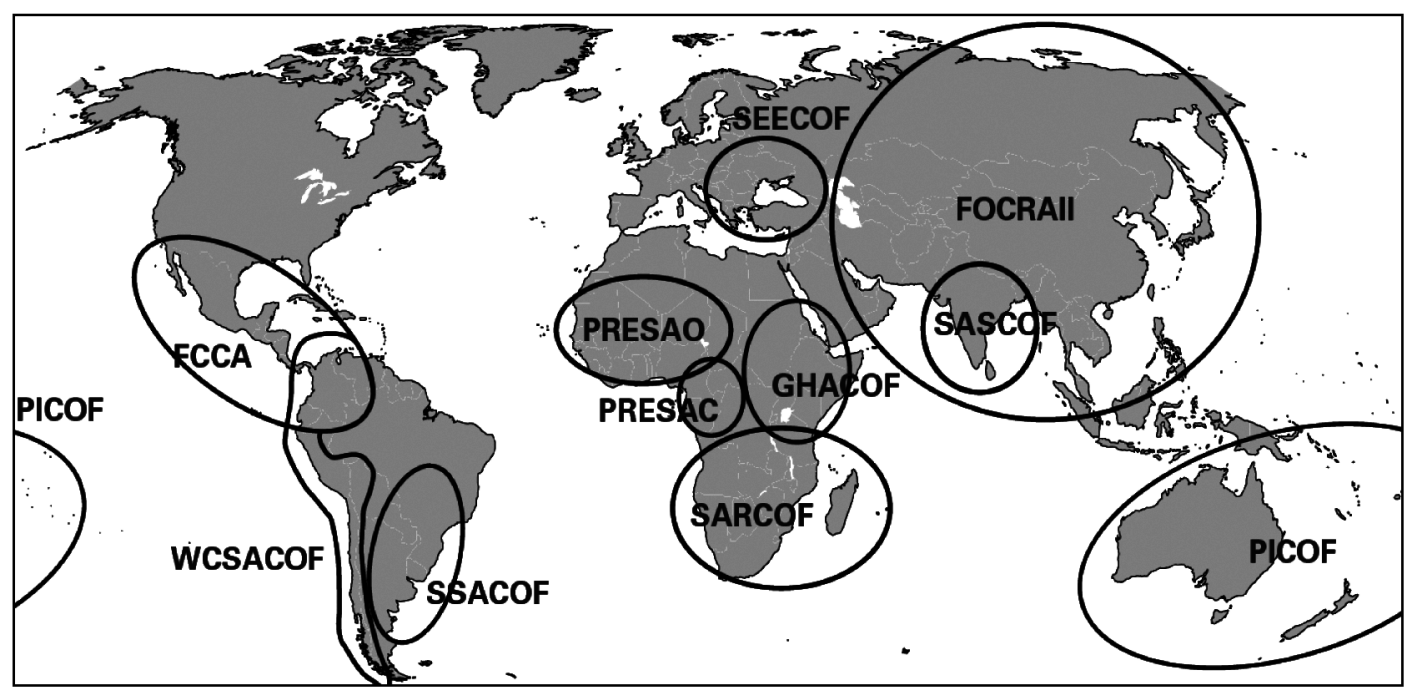

Fig. 1. Regional Climate Outlook Forums (RCOFs) operating worldwide. For complete list see Table 1

The WMO has helped establish RCOFs all over the world by recognizing that climate information, including predictions, could substantially benefit many climate-sensitive sectors in different parts of the world in adapting to and mitigating the impacts of climate variability and change. These forums have an overarching responsibility to produce and disseminate regional assessments (using a predominantly consensus-based approach) of the state of the regional climate for the upcoming season. However, the climate information products generated by the RCOFs still fall far short of end-user needs in terms of resolution, timing of the products for seasonal prediction, and the relevance of climate information products required by specific users. As a result, a major portion of the climate information generated for the RCOFs is not useable by application sectors. This problem is compounded further by the prevalence of a 'donor mentality' and the need to make a transformation to an 'owner mentality'. In a broader socio-economic context this problem has been analyzed by Lancaster (2003). Her analysis, which focuses on Africa, suggests a need for aid-donor agencies to empower donor-support recipients to become the owners of the development programs. She asserts that without building this capability, it is impossible to ensure that nations will be able to develop their own capacity for economic, social and political growth. This transformation is also needed for the development of climate science research and services in developing countries. We invoke this principal to motivate the proposed climate risk management strategies (CRMS) framework.

Although numerous research studies have been carried on the global climate through the Climate Variability and Predictability (CLIVAR) project of the
Table 1. Acronyms

\begin{tabular}{|ll|}
\hline Acronym & Definition \\
\hline CLIPS & Climate Information and Prediction Services \\
CLIVAR & Climate Variability and Predictabilty \\
COF & Climate Outlook Forum \\
CRMS & Climate Risk Management Strategies \\
FCCA & Central America COF \\
FOCRAII & Forum On regional Climate monitoring, \\
& assessment and prediction for Regional \\
GFCS & Association II (Asia) \\
GHA & Global Framework for Climate Services \\
GHACOF & Greater Horn of Africa COF \\
GPC & Global Producing Centers of Long Range \\
ICPAC & Forecasts \\
IDRC & Internate Prediction and Applications Center \\
KMA & Korea Meteorological Agency \\
KOICA & Korea International Cooperation Agency \\
NHMSs & National Meterological and Hydrological \\
NGOs & Services \\
PICOF & Pacific Islands COF \\
PRESAC & Central Africa COF \\
PRESAO & Western Africa COF \\
RCCs & Regional Climate Centers \\
RCOF & Regional Climate Outlook Forum \\
SARCOF & Southern African Regional COF \\
SASCOF & South Asian COF \\
SEECOF & Southeastern Europe COF \\
SSACOF & Southeast of South America COF \\
START & System for Analysis Research and Training \\
TF & CLIVAR-CLIPS Joint Task Force \\
WCRP & World Climate Research Program \\
WCSACOF & Western coast of south America COF \\
WMO & World Meteorological Organization \\
\hline
\end{tabular}




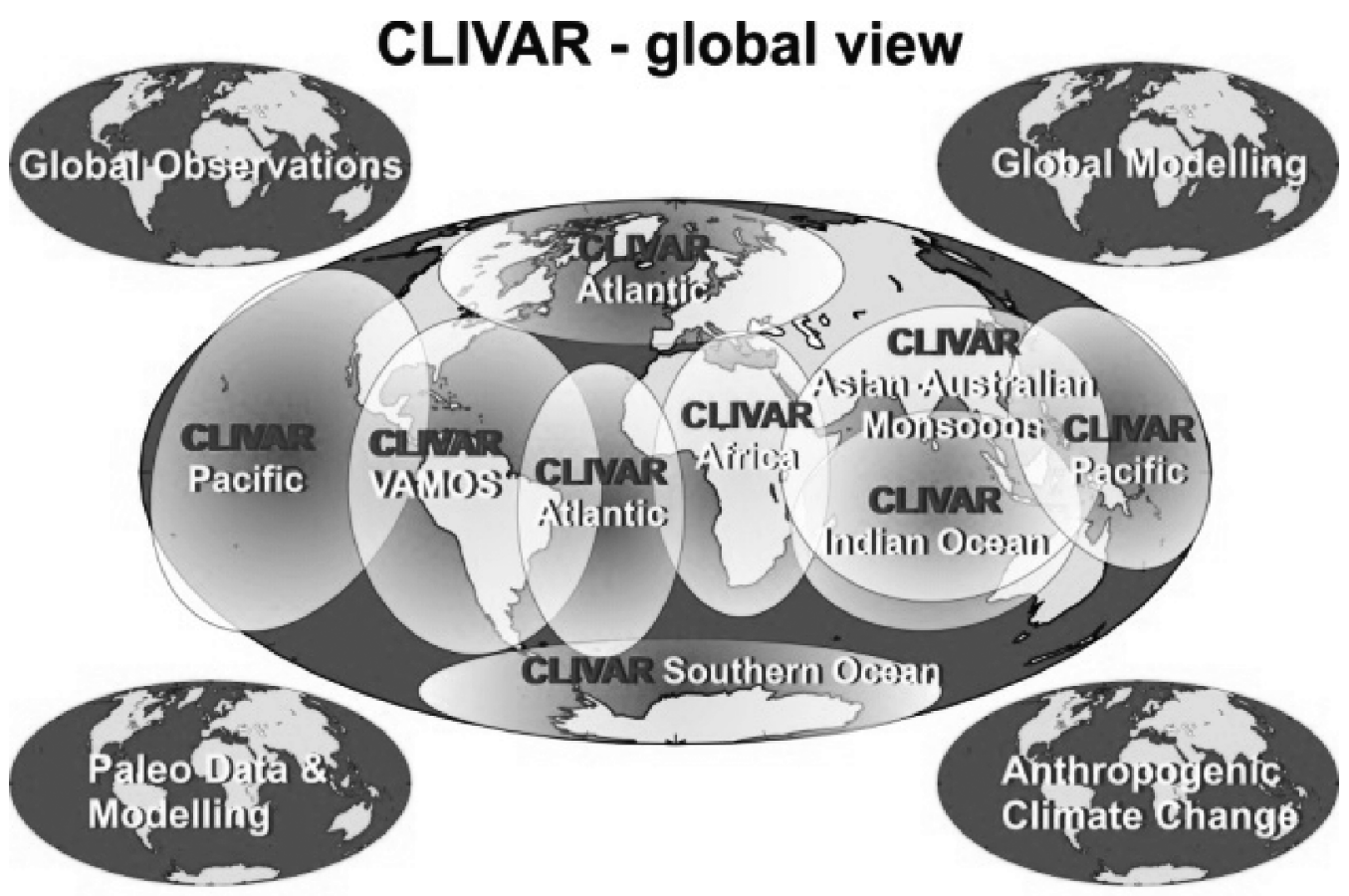

Fig. 2. World Climate Research Program's global CLIVAR (Climate Variability and Predictability) project (www.clivar.org)

World Climate Research Program (WCRP; Fig. 2), the impact of these advances on the socio-economic conditions in developing countries is still very limited. CLIVAR addresses the research on climate variability and predictability, with a particular focus on the role of ocean-atmosphere interactions in climate. It works closely with its companion WCRP projects on issues such as the role of the land surface, snow and ice, and the role of stratospheric processes in climate. A previous review of RCOF activities (RCOF 2008) and their recent 10 yr anniversary evaluation (GFCS 2010) identified several non-traditional areas of climate research required to support the integration of climate information into developmental planning for improving the performance of climate-sensitive sectors. These areas are closely aligned with the agenda for the CLIVAR project. It is apparent that combined guidance from CLIVAR for research and CCL for applications is needed.

As a result of the disconnection between research activity and climate services, a self-sustaining cycle is continuously eroding and undermining any progress. The cycle begins with poor support and funding for research and climate services. This results in the inability of climate service providers to meet stakeholder needs, which, in turn, results in the further erosion of support and the strengthening of the undesirable positive feedback process. In the present paper, we propose a framework aimed at helping climate services to provide institutions in developing countries to con- cretely demonstrate the benefits of climate research and climate services, thereby breaking the cycle and creating stakeholders who will use these climate services and advocate the funding of research and implement its results.

\section{TEMPLATE FOR CREATING A SUSTAINABLE FRAMEWORK FOR CLIMATE SERVICES}

To address these gaps, we propose an organizational structure and implementation strategy in the WMO/WCRP/GFCS system that will support a longterm sustainable program for the incubation of CRMS (Fig. 3) for decision-making in developing countries. The design of CRMS is aimed at supporting an effective end-user-centered communications strategy, including delivery, consultation and results monitoring. CRMS organizational structure and implementation strategy comprise the following components:

(1) RCOF-coordinating agencies or Regional Climate Centers (RCCs) serving as executing sites for CRMS incubation projects.

(2) CLIVAR-CLIPS joint task force (TF) for guiding relevant crosscut research.

(3) A WMO/WCRP/GFCS mechanism supported by climate change programs including START, IDRC and others to coordinate implementation aspects of the CRMS agenda. 


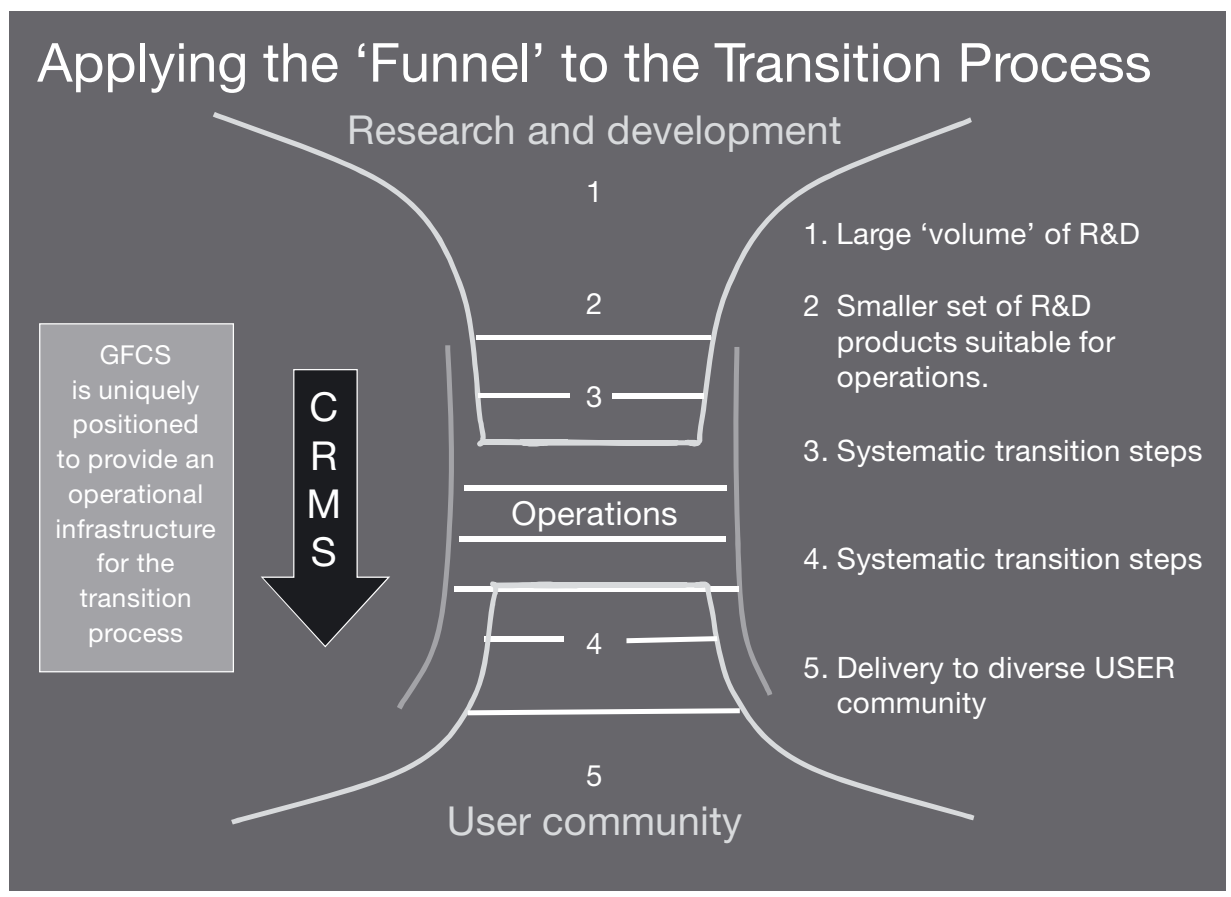

Fig. 3. Design of CRMS (Climate Risk Management Strategies) 'test-bed' (illustration adapted from the National Oceanic and Atmospheric Administration climate test bed framework, Vera \& Higgins 2006). GFCS: Global Framework for Climate Services

\section{IMPLEMENTATION SITES FOR THE CRMS AGENDA}

The mission of the proposed CRMS research program is to create a capacity building platform for a dedicated multidisciplinary community, of university, industry and government partnerships to design, test and introduce climate risk management strategies for a greater quality of life in the regions served by the CRMS program and its hosting institutions.

The natural implementation sites for the CRMS agenda are the RCOF-coordinating agencies or RCCs in developing countries. We envisage that this will entail formation of interdisciplinary teams of 5 to 10 people working together continuously from a few months up to a year at a relevant institution dealing with regional climate to develop practical strategies for climate risk management. Members of this team would come from advanced centers and universities in developed and developing countries, including participants from NMHSs, RCC staff and representatives of the application sectors. The goal is that the outcomes generated by these teams would be migrated to the mainstream operational RCC environment in a much shorter period of time than would otherwise have been possible. The expectation is that the high yield strategies produced by these teams will encourage developing nations to invest in climate science research by increasing and sustaining support for in-country research activities for managing climate risk and increasing the performance of climate-sensitive socio-economic sectors. This would have the important effect of breaking the present 'lowvalue, low-use, low-research' support 'cycle' through concrete demonstration of the value of climate services and, therefore, promoting an atmosphere conducive for sustained funding, and for support of further investment in this enterprise.

A CRMS should have 3 primary attributes: (1) fostering interdisciplinary research and assessment synthesis, (2) improving our understanding of and bridging the gap between climate science research and applications, and (3) contributing to regional decision support and climate services. Other attributes should include:

- Provision of pathways for the GFCS's integration of adaptation actions into development plans in developing countries

- Provision of streams of valuable feedback for evaluating added value of the model prediction products. The CRMS should support the development of sustainable partnerships between universities, research institutions and RCCs, to accelerate the assimilation of research into operational climate services

- The CRMS should support integrated, location-relevant research for decision makers' options in the face of climate change and other stressors at the regional 
level. This should be accomplished in a manner that is cognizant of the context of the decision-makers and within the constraints they face in managing their climate-sensitive resources

- The CRMS should promote and exploit innovative and embedded long-term partnerships among a spectrum of interested parties including national, local and private entities

- The CRMS should promote partnerships among institutions focusing on the region and build on existing efforts within the region to study the impacts of climate change in support of climate services

- CRMS efforts must: (1) involve stakeholders in the design and assessment of the research, and (2) develop and communicate innovative and transferable methods, knowledge, information, and/or tools for understanding and adapting to the changing climate. This approach should serve as a mechanism for the creation, dissemination and exchange of climaterelated research findings and decision-support resources critical for understanding and addressing resource management challenges in user sectors.

\section{CLIVAR-CLIPS TASK FORCE}

The terms of reference (TORs) for the proposed CLIVAR-CLIPS TF could be based on the joint statement issued by the experts representing the WCRP and the WMO CCL, who met in a joint session on 18 February 2010 in Antalya, Turkey. The full statement is available at CCL-WCRP (2010).

\section{CRMS COORDINATION MECHANISM}

The CRMS initiative will require a dedicated coordination mechanism for its implementation activities. In particular, the mechanism will serve to implement the decisions and recommendations made by the proposed CLIVAR-CLIPS TF. Secondly, administrative coordination is required for streamlining the presently uncoordinated but highly complementary climate-application projects. Many of these projects are, in essence, incubation projects for the purpose of developing capacity to use climate prediction and projections information to enhance the performance of climate-sensitive sectors.

However, these projects are of short-term duration, and their intended impacts could diminish with time. The CRMS approach will, therefore, play a critical role in promoting long-term sustainability for these demonstration projects, focusing on the integration of climate science into developmental planning actions for climate-sensitive socio-economic sectors. As examples we highlight some of the key attributes of 2 recent lim- ited-time collaborative projects for the eastern Africa region that would be good candidates for implementation under the proposed CRMS agenda to promote long-term sustainability.

The Korea Meteorological Agency/Korea International Cooperation Agency (KMA/KOICA) project serves to strengthen and advance the capability of the ICPAC (Climate Prediction and Applications Center) as a central hub of climate monitoring, state-of-the-art-modeling, prediction and downscaling for the eastern Africa region, networked with regional NMHSs through an interactive operational framework. The duration of the project is planned for about $2 \mathrm{yr}$, and its focus is to help in developing a capacity in the region to provide highquality, high-resolution and reliable regional climate change products and services. It is also designed to help facilitate the integration and optimize the understanding and use of the above-mentioned information within the various user communities that are vulnerable to climate variability and the risks associated with climate change. The project is being implemented by the ICPAC, coordinated by the WMO in close collaboration with the NMHSs of the Greater Horn of Africa and it involves the following countries: Burundi, Ethiopia, Eritrea, Kenya, Somalia, Sudan, Tanzania and Uganda. The second example, which is also implemented by the ICPAC, coordinated by the WMO and planned to run for ca. $2 \mathrm{yr}$, is funded by the World Bank and it is complimentary to the project outlined in the first example but with a slightly different focus of providing hands-on experience to the regional experts in handling climate change projection products. It is reasonable to expect that under the proposed CRMS framework the 2 projects could have benefited from its 3-component structure and combined to yield more tangible and long-lasting outcomes. The coordination and implementation components were consistent with our proposed framework for CRMS but input from the crosscut research community (CLIVAR-CLIPS TF) was entirely missing.

We have chosen these 2 examples to highlight some of the benefits of the CRMS approach. These initiatives are already making a critical contribution in capacity building and research to support climate services in eastern Africa, but we believe the returns could be significantly increased through the proposed CRMS approach. Numerous other projects supported by major organizations in developing countries have very limited or no coordination among them, resulting in non-optimal outcomes and expectations. The proposed CRMS project would provide a global framework for long-term sustained coordination of such activities. The specific benefits that would arise from the seamless coordination among the 3 components of the CRMS process described earlier are: 
- CLIVAR-CLIPS TF: This would provide continuous, unified and high-level science oversight currently based on relatively temporary strategies for science guidance - CRMS coordination mechanisms: This component would help to optimize the leveraging of resources for demonstration projects, coordinate the mobilization of funding, provide centralized prioritization and coordination of the demonstration projects to avoid the unnecessary duplication of work

- Implementation sites: This is the delivery arm of the enterprise and the primary outlet to the end-users of CRMS products. Their ability to deliver high-level products will be the primary asset for the creation of a dedicated community of locally based advocates and sources of funding for its products.

The combined result would be an enhanced sustainability of high-quality climate services and research, which has been an illusive goal for many years in developing countries.

\section{HUMAN RESOURCES AND CRMS PROJECTS}

Expertise will be required in regional dynamical climate modeling, statistical modeling, computing and information technology, web-site development and social communication. Besides having dedicated staff at regional institutions, such as RCCs, an important element of human capacity would be visiting faculty consisting of 'interdisciplinary teams'. These special teams of 5 to 10 experts would be comprised of researchers from developed and developing countries and national representatives from concerned countries in the region, who would be responsible for the development and testing of new strategies for the provision of regional or in-country climate services. A successful CRMS program will require creation of a sustained program for exchange visits of experts between partner institutions in developed countries and regional institutions, including RCCs, with education as its key focus, through the CRMS incubation activities. Examples of potential CRMS projects include: - Seasonal forecasting ensemble prediction methodologies based on input from the 11 designated GPCs (Global Producing Centers of long-range forecasts) in RCOF tercile format and other forms optimized for climate-information-user forums

- Modernization of the traditional RCOF forecasting model and blending it with GPC-based experimental prediction products for operational purposes

- Development of climate risk management products for a broad range of RCC stakeholders

- Development of self-configurable RCM-based downscaling systems to facilitate appropriate use of RCMs

- Development of virtual RCOF alternatives to the traditional RCOF approach involving physical meetings.

\section{CONCLUSIONS}

We have proposed a new mechanism that can break the cycle inhibiting growth in climate services in developing countries. We have proposed a structure that exploits existing capabilities for the development of CRMSs to support climate services in developing countries. This mechanism is comprised of 3 components, namely, implementation sites for the CRMS incubation projects, a CLIVAR-CLIPS TF for guiding the relevant crosscut research and a WMO/WCRP/GFCS mechanism for coordinating the implementation of the CRMS agenda.

CRMS would have to manage a highly diverse portfolio of projects. Careful thought must be given to the formulation of its responsibilities to ensure efficiency and maximum impact. A key role of CRMS is to distill the common synergisms among the diverse demonstration projects involved in climate change research to ensure that the lessons learned benefit all of the projects and avoid repetition in order to minimize costs while consolidating long-term sustainability. Furthermore, it should not be the responsibility of CRMS to manage all aspects of the demonstration projects. Rather, the science managers of the donor organizations would be expected to continue managing those aspects of the incubation projects they fund that do not need centralized coordination and are not critical for promoting project-to-project cross-pollination. Once these projects mature, the RCCs and local support would be in a position to continue these activities without the support of external multilateral aid agencies. Attention would then be directed towards new areas of climate services and research that need start-up resources and expertise for incubation projects.

Acknowledgements. We thank the anonymous reviewers who helped to improve the paper.

\section{LITERATURE CITED}

CCL-WCRP (Commission for Climatology-World Climate Research Program) (2010) Statement, Antalya, Turkey, 18 February 2010. Available at: www.wmo.int/pages/prog/ wcp/ccl/documents/Joint_CCl_WCRP_Statement_2010.pdf (accessed 26 September 2010)

CLIPS (Climate Information and Prediction Services) (2010) Climate Information and Prediction Services (CLIPS) Project. Available at: www.wmo.int/pages/prog/wcp/wcasp/ CLIPSIntroduction.html (accessed 26 September 2010)

GFCS (Global Framework for Climate Services) (2010) High level taskforce toward the Global Framework for Climate Services (GFCS). Available at: wmo.int/hlt-gfcs/index_en.html

Lancaster C (2003) Aid to Africa. University of Chicago Press, Chicago, IL

RCOF (Regional Climate Outlook Forum) (2008) RCOF review 2008. Available at: www.wmo.int/pages/prog/wcp/wcasp/ RCOFReview2008.html (accessed 26 September 2010)

Vera C, Higgins W (2006) Variability of the American monsoon systems, Chair's Repüort, VPM9. www.clivar.org/ organization/vamos/VPM9\%20presentations/Vera_Higgins_ Chairs.report.18apr06.pdf 